Bull. Mater. Sci., Vol. 39, No. 7, December 2016, pp. 1671-1676. (C) Indian Academy of Sciences.

\title{
Morphological and optical properties of n-type porous silicon: effect of etching current density
}

\author{
M DAS and D SARKAR* \\ Department of Physics, Gauhati University, Guwahati 781014, India
}

MS received 9 March 2015; accepted 10 May 2016

\begin{abstract}
Morphological and optical properties of porous silicon (PS) layer fabricated on n-type silicon wafer have been reported in the present article. Method of PS fabrication is by photo-assisted electrochemical etching with different etching current densities $(J)$. Porosity and PS layer thickness, obtained by the gravimetric method, increase with increasing $J$. Pore morphology observed by FESEM shows the presence of randomly distributed pores with mostly spherical shape. Calculated pore size is also seen to increase with increasing value of $J$. XRD gives the characteristic amorphous peak of PS along with some peaks corresponding to crystalline silicon (c-Si). Calculated crystallite size shows decreasing trend with increasing $J$ value. The optical properties of these samples have been investigated by UV-visible reflectance, Raman spectroscopy and photoluminescence (PL) spectra. Reflectance measurement shows blue-shift of the spectrum with increased reflectivity for increasing $J$. Raman spectra show remarkable blue-shift with respect to the c-Si peak. PL spectra give the luminescence energy in the orange-red region of the visible spectrum and little change with variation of $J$.
\end{abstract}

Keywords. Porous silicon; anodization; reflectance; photoluminescence; Raman spectra.

\section{Introduction}

Though silicon has been the dominant material for electronics, its applicability in optics has been limited by its indirect bandgap nature [1-3]. However advent of porous silicon (PS) changed this notion appreciably. Observation of room temperature visible photoluminescence (PL) $[4,5]$ in PS has demonstrated its applicability in optoelectronics applications similar to that of direct gap semiconductors. PS has been reported to exhibit large variety of morphology and its luminescence property is ascribed to the quantum confinement effect [6], i.e., somehow or other related to morphology, pore size, porosity, etc. Though extensive research in this direction has been carried out, the exact luminescence mechanism and related nanostructures still remain unclear [7] although Deng et al [8], based on their current findings, have discussed some efforts to advance the PL application of silicon nanocrystals. Hence, a further study on this is still needed. PS can be easily prepared by electrochemical etching popularly known as anodization, first described by Uhlir [9] more than five decades ago. However, for n-type silicon, irradiation of the anodization bath is required [10] for homogeneous pore distribution. By changing the formation parameters, viz., etching current density, electrolyte concentration, anodization bath temperature [11], irradiation wavelength/intensity, etc., PS with large variety of morphology and porosity can be produced, which in turn will change the optical properties [12]. Behzad et al [13] investigated the effect of etching time and

\footnotetext{
*Author for correspondence (sarkardeepali@gmail.com)
}

etching current density on physical, thermal and optical properties and found that thermal diffusivity and bandgap of the PS increase with increasing porosity. However, Cho et al [14] reported that surface morphology of PS prepared at current density below $200 \mathrm{~mA} \mathrm{~cm}^{-2}$ exhibited very stable and even surface but displayed cracked surface when prepared at current density above $300 \mathrm{~mA} \mathrm{~cm}^{-2}$. On the other hand Sheng et al [15] reported that optical bandgaps of p-type PS are higher than that of n-type PS. For in-depth understanding, correlation of observed physical properties with morphology of PS films and preparation parameters is very much in need. In the present investigation we attempt to show interrelation between morphology and optical property, with one fabrication condition, etching current density of n-type silicon. In particular, we report here FESEM for surface morphology, XRD for crystallinity and crystallite dimension, and UV-visible reflectance, Raman spectra and PL for optical properties of n-type PS prepared by changing the anodization current density for four different values keeping all the other preparation parameters constant.

\section{Experimental}

The PS samples are prepared by photo-assisted electrochemical etching of (100)-oriented phosphorus-doped crystalline silicon (c-Si) wafer of resistivity $\sim 1-10 \Omega \mathrm{cm}$ and thickness $380 \pm 25 \mu \mathrm{m}$. Before the etching process, the c-Si wafers are pre-treated with HF and ethanol in an ultrasonic bath for $5 \mathrm{~min}$ to remove any native oxide present on the silicon surface [16]. The electrochemical cell used is an indigenous 
two-electrode one with surface area of $4.53 \mathrm{~cm}^{2}$. Platinum coil is used as a cathode and c-Si back-contacted with aluminum foil as the anode. The electrolyte used is a mixture of $(48 \%) \mathrm{HF}: \mathrm{C}_{2} \mathrm{H}_{5} \mathrm{OH}(99.9 \%)$ taken in a volumetric ratio of 1:1. Electrochemical etching is carried out at four different values of anodization current densities $(J)$ : 13.2, 17.6, 22.1 and $26.5 \mathrm{~mA} \mathrm{~cm}^{-2}$, for a constant etching time duration of 30 min using current source from a dc power supply (agilent-6634B). Henceforth these four PS samples obtained for four different etching current densities will be referred to as I, II, III and IV, correspondingly. It is customary to etch n-type silicon under illumination as pore formation in silicon is supposed to be due to excess holes present in the material; in n-type one they are minority carriers; hence one has to increase the number by some external source. The etching is performed under front side illumination with a 200-W UV illuminator source (Newport 69901 UV illuminator). After anodization, the samples are rinsed several times with ethanol and then dried and stored under vacuum. Porosity (\%) and thickness of the prepared PS films are measured by the gravimetric method, which is very simple in its nature but at the same time is very effective. FESEM pictures for morphology study are recorded by a SEM-FEI NOVA NANOSEM 450. XRD for structure and crystallinity study are recorded by a D-8 Advance X-ray diffractometer. Optical properties of the samples are studied through measurement of reflectance spectra, Raman spectra and PL spectra. Optical reflectance spectra are recorded using a carry-300 scan UV-visible spectrophotometer through a wavelength range of 360-800 nm. Raman spectra are recorded using a JY Horiba T6400 micro-Raman set-up for excitation wavelength of $425 \mathrm{~nm}$. Room temperature PL spectra are recorded by a fluorescence spectrophotometer (Varian Cary Eclipse) for the excitation wavelength of $425 \mathrm{~nm}$.

\section{Results and discussion}

At the very outset the porosity and thickness of the obtained porous layer on n-type silicon have been determined as these are the basic parameters that dictate morphological, structural and optical properties of PS. Porosity $(P)$ and thickness $(T)$ of the PS samples are estimated from gravimetric measurement [17] using the equations

$$
\begin{aligned}
& P(\%)=\left(m_{1}-m_{2}\right) /\left(m_{1}-m_{3}\right), \\
& T(\mu \mathrm{m})=\left(m_{1}-m_{3}\right) / A \delta_{\mathrm{Si}},
\end{aligned}
$$

where $m_{1}$ and $m_{2}$ are the masses of Si-wafer before and after PS layer formation, respectively, and $m_{3}$ is the mass of Siwafer after PS removal by etching in $1 \% \mathrm{KOH}$ solution. $A$ is the area of the PS layer and $\delta_{\mathrm{Si}}$ the density of silicon wafer. Porosity value, calculated by this method, is found to range from 55 to $78 \%$ while the thickness is from 25 to $34 \mu \mathrm{m}$ in the samples I-IV. This ascertains clear indication of increase of both these parameters with increase in etching current density. To have better visual guidance, these parameters are plotted $v s$. the etching current density $(J)$ and also listed in tabular form. They are depicted in figure 1 and table 1.

Morphology of the PS samples has been studied through FESEM images. In figure 2 we show the images of the samples I-IV. The pores are seen to be randomly distributed on the surface. It is clear from these pictures that the pores are mostly spherical in shape with no significant dependence of pore shape on $J$. However, mean pore size $(<d>$ ), obtained from the particle size-frequency histogram of the FESEM images (shown in the inset of the FESEM images) is 63, 84, 94 and $100 \mathrm{~nm}$, respectively, for samples I, II, III and IV. Thus the pore size is also seen to increase with increasing $J$ value. In table 1 we quote these values too along with porosity $(P)$ and pore thickness $(T)$.

In figure 3 we show the XRD patterns of these PS samples in the incident angle range of $2 \theta=15-75^{\circ}$. The broad hump at around $2 \theta=22^{\circ}$ is attributed to the amorphous nature of

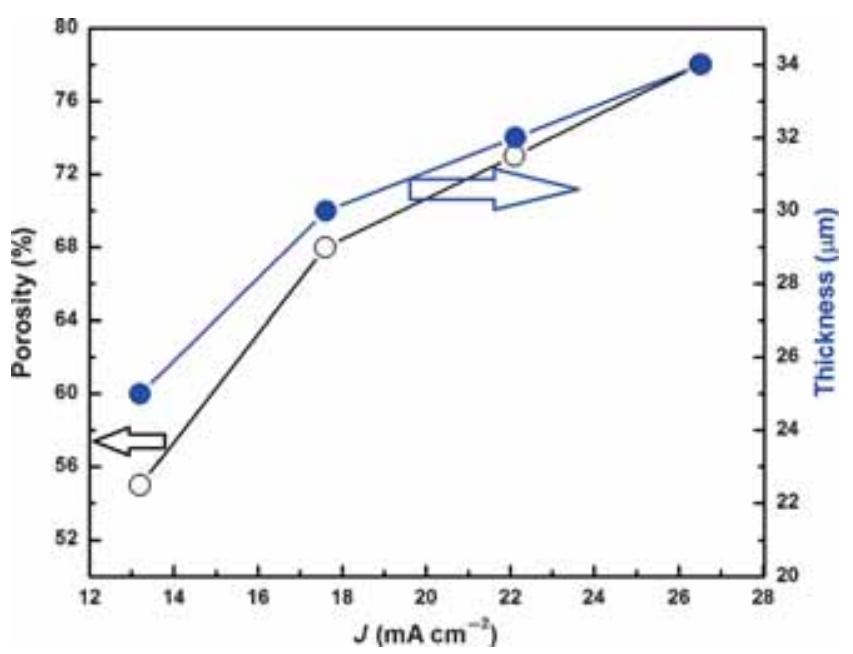

Figure 1. Porosity $(\%)$ and thickness $(\mu \mathrm{m}) v s$. etching current density $(J)$ of the PS samples.

Table 1. Porosity $(P)$, layer thickness $(T)$, mean pore size $(<d>)$, effective refractive index $(n)$, crystallite size $(L)$, and Raman and PL

\begin{tabular}{|c|c|c|c|c|c|c|c|c|}
\hline Sample & $\begin{array}{l}\text { Current density } \\
\left(\mathrm{J} \mathrm{mA} \mathrm{cm}^{-2}\right)\end{array}$ & $P(\%)$ & $T(\mu \mathrm{m})$ & $n$ & $<d>(\mathrm{nm})$ & $L(\mathrm{~nm})$ & Raman peak $\left(\mathrm{cm}^{-1}\right)$ & PL peak $(\mathrm{nm})$ \\
\hline I & 13.2 & 55 & 25 & 1.9 & 63 & 23 & 504 & 630 \\
\hline II & 17.6 & 68 & 30 & 1.6 & 84 & 21 & 502 & 625 \\
\hline III & 22.1 & 73 & 32 & 1.5 & 94 & 19 & 500 & 621 \\
\hline IV & 26.5 & 78 & 34 & 1.4 & 100 & 13 & 498 & 615 \\
\hline
\end{tabular}
peak positions for PS samples. 

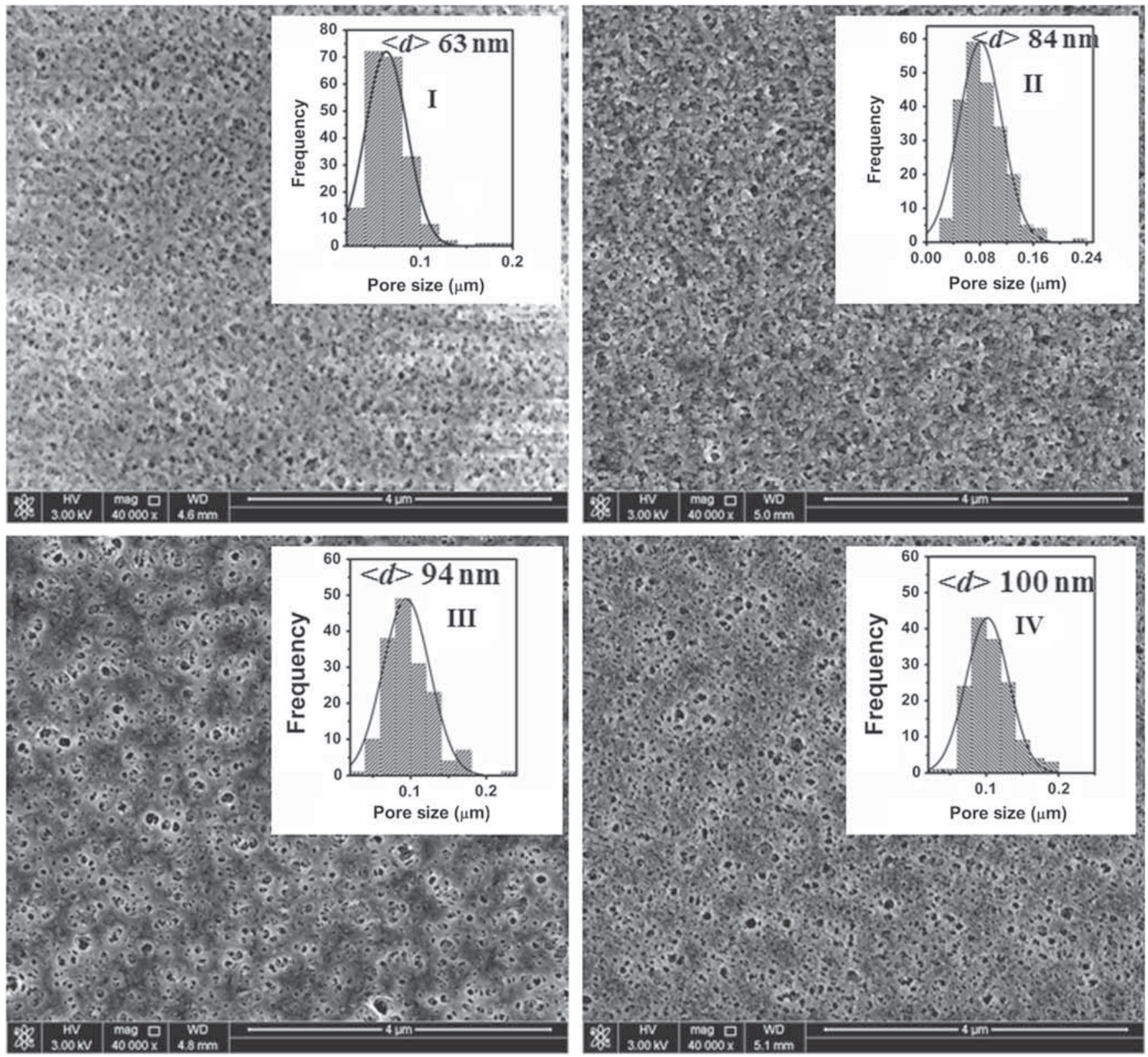

Figure 2. FESEM micrographs of the four PS samples.

PS, which arises due to the exposure of PS to the atmosphere and has been caused due to impregnation of atmospheric oxygen [18]. The weak peaks at $2 \theta=33^{\circ}$ and $61.7^{\circ}$ correspond to the (200) and (320) reflection planes of c-Si, respectively $[19,20]$. The strong peak at $2 \theta=69.1^{\circ}$ corresponds to the first allowed (400) reflection from (100)-oriented c-Si. However, on PS formation the full-width at half-maximum (FWHM) of this peak increases from $0.43^{\circ}$ to $0.75^{\circ}$ as the etching current density is increased, that is, as we move from sample I to sample IV. This is a clear indication of decrease in Si crystallite size in PS with increasing etching current density. FWHM of (400) peak is also estimated from the Gaussian fitted curve. Si crystallite size $(L)$ has been calculated from this (400) peak using the well-known Scherrer equation [21], $L=0.9 \lambda / \beta \cos \theta$, where $\beta$ is FWHM of the peak.
Calculated $L$ values for the PS samples are also listed in table 1 for instant comparison. Si-crystallite size is seen to decrease from 23 to $13 \mathrm{~nm}$ as we increase the etching current density from 13.2 to $26.5 \mathrm{~mA} \mathrm{~cm}^{-2}$.

In figure $4 \mathrm{a}$ we show the reflectance spectrum of these samples for the beam of light incident at an angle of $8^{\circ}$ on to the wafer. The interference pattern type of the spectrum indicates that the PS sample behaves like an interferometer. Such behaviour in PS has been shown in some of the earlier works also [22,23]. Along with this, also to be noted is the fact that with increase in etching current density from $J=13.2$ to $26.5 \mathrm{~mA} \mathrm{~cm}^{-2}$ the interference pattern as a whole shows shift towards lower wavelength side with increased intensity. This type of shifting of interference pattern is attributed to decrease of effective refractive index of the PS layer, which 
in turn is linked to increase in porosity [24,25]. Effective refractive indexes of the PS samples are calculated using the Bruggeman effective medium approximation [26,27]. Calculated refractive index $(n)$ values are also listed in table 1. The value of $n$ is seen to decrease from 1.9 to 1.4 as the porosity is increased from 55 to $78 \%$ with increasing etching current density. In figure $4 \mathrm{~b}$ we show a schematic diagram for reflectance mechanism, which shows reflection of light from the two interfaces in a PS sample, i.e., interference occurs between the reflected beams from the upper and the lower interfaces; thus we can say that $\lambda_{\min }$ and $\lambda_{\max }$ in the reflectance spectrum are observed due to destructive and

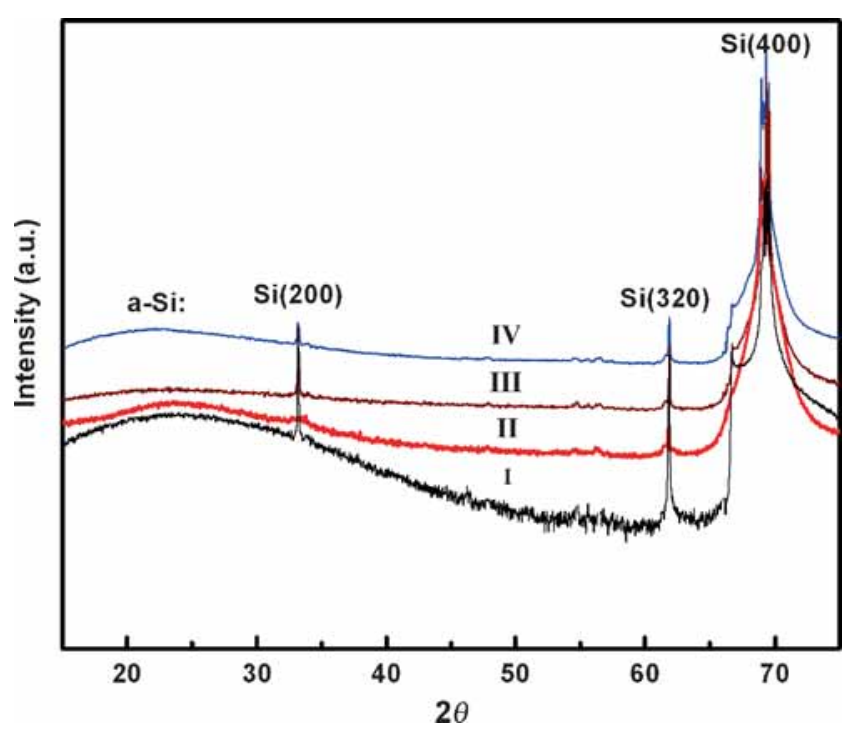

Figure 3. XRD pattern of the PS samples I-IV. constructive interference of the reflected light from the two interfaces.

To explore more about the optical properties of these samples we carried out the Raman study of these samples. In figure 5 we show these spectra of c-Si and PS samples in a and $b$, respectively. The Raman spectrum of $\mathrm{c}-\mathrm{Si}$ is peaked at $522 \mathrm{~cm}^{-1}$ with FWHM value of $4.8 \mathrm{~cm}^{-1}$. Raman peak position for PS samples is seen to lie in the range of 504$498 \mathrm{~cm}^{-1}$. Thus there is a large blue-shift with respect to c-Si (by $18 \mathrm{~cm}^{-1}$ ) and smaller blue-shift (by $2 \mathrm{~cm}^{-1}$ ) from one PS sample to the successive one with increasing etching current density. This type of shifting of Raman peak has been suggested to be due to confinement of optical phonons [28], which indicates the presence of small Si crystallites amongst the pores. These data are also in strong correlation to the XRD results discussed earlier, which indicate decrease of crystallite size with increase of etching current density.

Optical property study of PS is never complete without the study of PL. PL spectra of these samples are shown in figure 6. PL peak maxima for these samples are seen to have blue-shifted as $J$ is increased from 13.2 to $26.5 \mathrm{~mA} \mathrm{~cm}^{-2}$ with gradually reduced intensity. This type of blue-shift might be related to S-band emission [29] from PS. However these shifts are nominal as can be visualized from the corresponding energy values of 1.973, 1.989, 2.002 and $2.021 \mathrm{eV}$, respectively, for samples I, II, III and IV. The light emission in this type of materials generally has been established to come primarily from quantum confinement in nano-cSi domains formed during the etching process [30-32]. An explanation for such type of peak shift is given by the quantum confinement model [33]. According to this model, PL emission is due to band-band transition and is dependent on the size of Si crystallite in a way such that if the crystallite
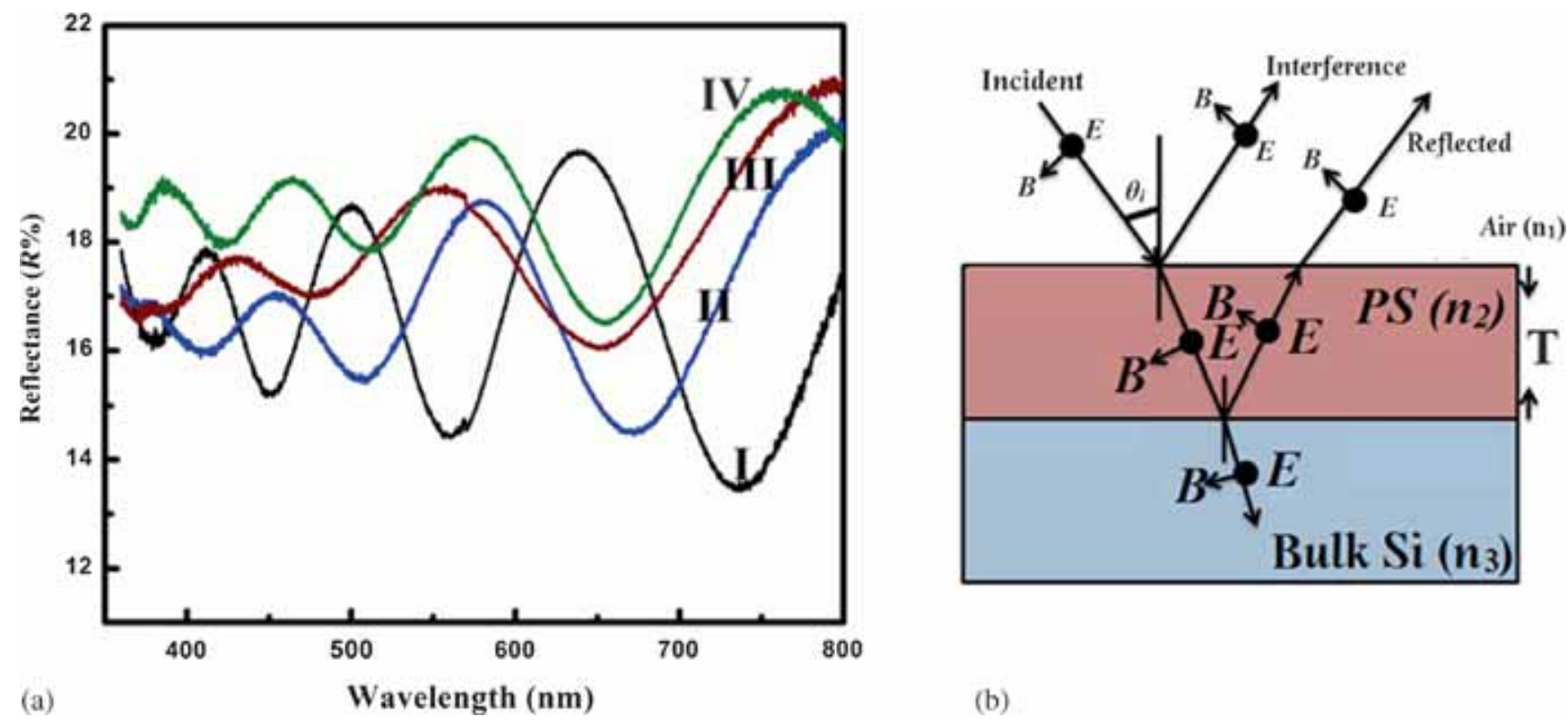

Figure 4. (a) Reflectance spectra of the samples I-IV and (b) schematic diagram of reflectance mechanism. 

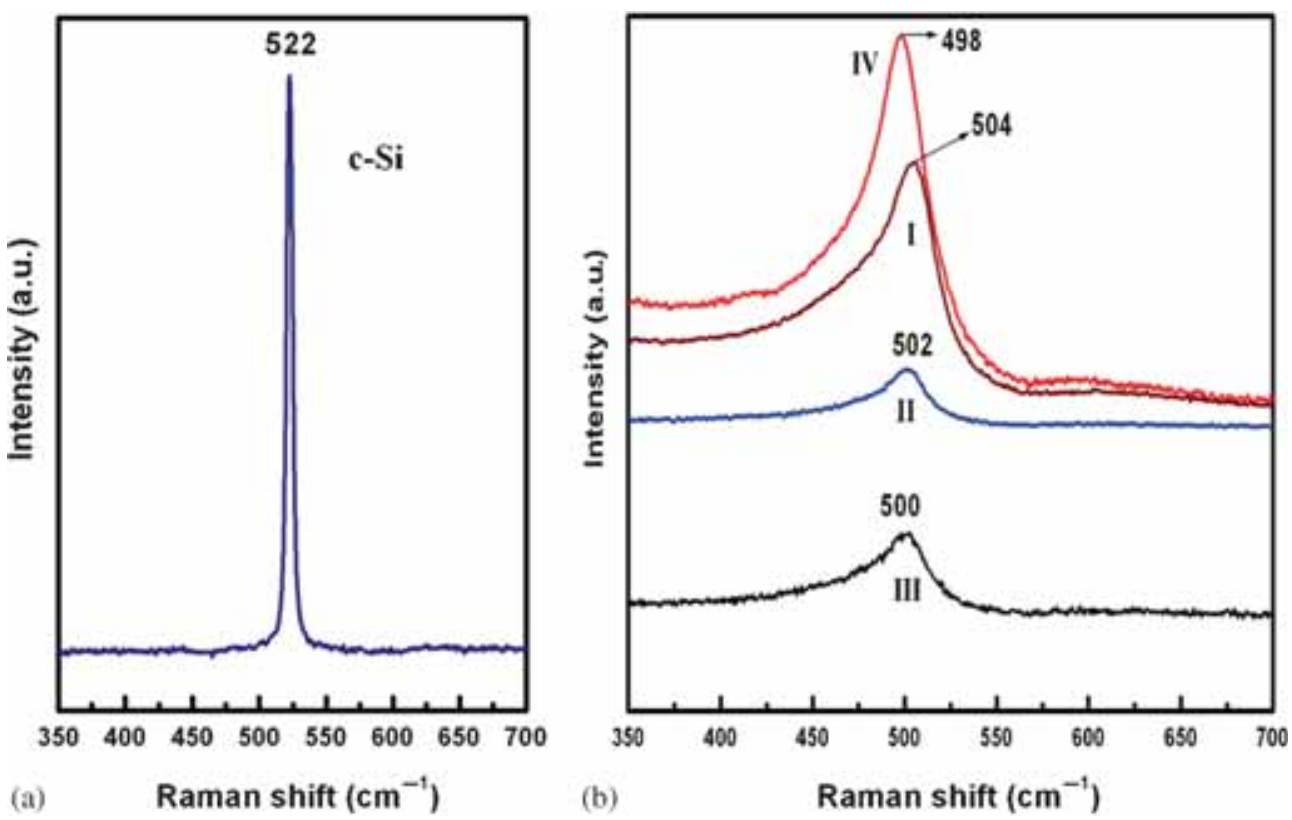

Figure 5. Raman spectra of (a) c-Si and (b) the PS samples I-IV.

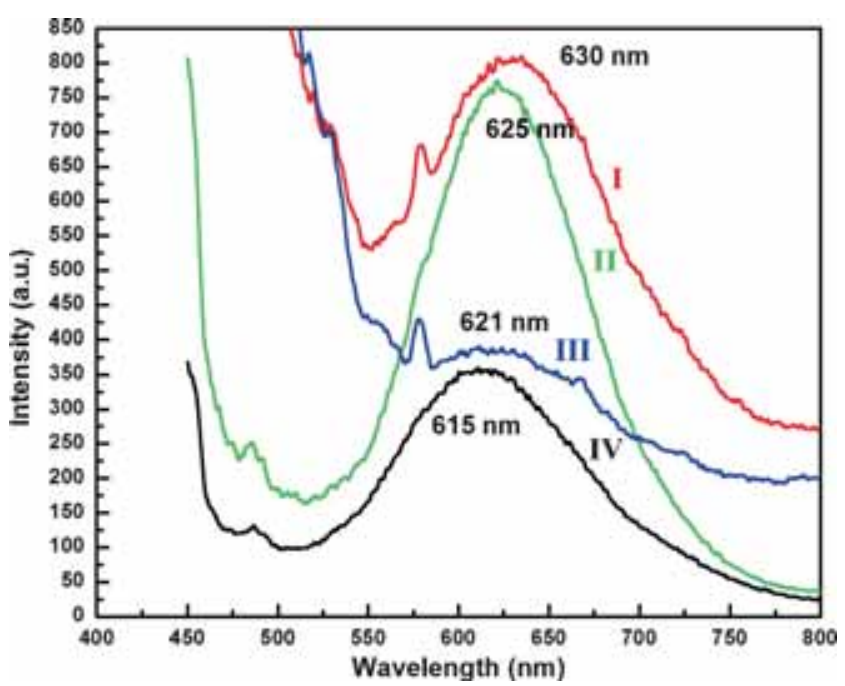

Figure 6. PL spectra of the samples I-IV.

size is smaller the emission peak is shifted to shorter wavelength. Raman and PL peak positions have also been listed in table 1 along with other results to facilitate ready comparison of the results of all the studies.

\section{Conclusions}

Porous silicon (n-type) samples were prepared for different anodization current densities using photo-assisted electrochemical etching and the effect of etching current density on surface morphology, reflectance, Raman spectra and photoluminescence (PL) was observed. From FESEM microscopy we observe that increased current density increases the pore size but has almost no effect on the pore shape. From XRD we observe that crystallinity decreases as we increase the etching current density. Reflectance spectra show a multireflection pattern similar to that of an interference plate with gradual blue-shift of the pattern with increasing etching current density and also with increasing intensity. Raman spectra and PL of the samples also indicate quantum confinement behaviour in small c-Si crystallites in between the porous structures. Thus the preparation parameter, viz., etching current density, is seen to have remarkable effect on porosity, surface morphology and crystallinity and this effect in turn is strongly correlated to optical properties through reflectance, Raman spectra and PL.

\section{Acknowledgements}

We are thankful to the Department of Science and Technology (DST) for its financial support through the project DST/TSG/PT/2009/96 and to IACS Kolkata for XRD and Raman measurements.

\section{References}

[1] Canham L T 1990 Appl. Phys. Lett. 571046

[2] Torres Costa V, Martin Palma R J and Martinez Duart J M 2004 J. Appl. Phys. 964197

[3] Dubey R S and Gautam D K 2011 Superlattices Microstruct. 50269

[4] Lehmann V and Gosele U 1991 Appl. Phys. Lett. 58856

[5] Halliday D P, Holland E R, Eggleston J M, Adams P N, Cox S E and Monkman A P 1996 Thin Solid Films 276299

[6] Lockwood D J and Wang A G 1995 Solid State Commun. 94 905 
[7] Kim D A, Im S I, Whang C M, Cho W S, Yoo Y C, Cho N H, Kim J G and Kwon Y J 2004 Appl. Surf. Sci. 230125

[8] Deng Z, Pi X D, Zhao J J and Yang D 2013 J. Mater. Sci. Technol. 29221

[9] Uhlir A 1956 The Bell System Technical Journal 35333

[10] Xiaopeng Li, Hong-Seok Seo, Han-Don Um, Sang-Won Jee, Yong Woo Cho, Bongyoung Yoo and Jung-Ho Lee 2009 Electrochim. Acta 546978

[11] Rump K, Granitzer P, Polt P, Reichmann A and Krenn H 2006 Thin Solid Films $\mathbf{5 1 5} 716$

[12] Dian J, Macek A, Nizansky D, Nemele I, Vrkostav V, Chovojka T and Jelinek I 2004 Appl. Surf. Sci. 238169

[13] Kasra Behzad, Wan Mahmood Mat Yunus, Zainal Abidin Talib, Azmi Zakaria and Afarin Bahrami 2012 Int. J. Electrochem. Sci. 78266

[14] Cho B, Jin S, Lee B Y, Hwang M, Kim H C and Sohn H 2012 Microelectron. Eng. 8992

[15] Chan Kok Sheng, Mahmood Mat Yunus W, Wan Md. Zin Wan Yunus, Zainal Abidin Talib and Anuar Kassim 2008 Physica B 4032634

[16] Sailor M J 2012 Porous silicon in practice: preparation, characterization and application (Germany: Wiley-VCH Verlag $\mathrm{GmbH} \& \mathrm{Co} . \mathrm{KGaA}$ ) p 51 Hoboken, New Jersey

[17] Sharma S N, Bhagvannarayan G, Sharma R K and Lakshmikumar S T 2006 Mater. Sci. Eng. B 127255

[18] Prabakaran R, Raghavan G, Tripura Sundari S, Kesavamoorthy R and Francis Xavier P 2002 Physica E 15243
[19] Kim H and Cho N 2012 Nanoscale Res. Lett. 7408

[20] Das M and Sarkar D 2013 Indian J. Pure Appl. Phys. 51724

[21] Cullity B D 1959 Elements of X-ray diffraction (London: Addison-Wesley) p 110

[22] Janshoff A, Dancil K-P S, Steinem C, Greiner D P, Lin V S-Y, Gurtner C, Motesharei K, Sailor M J and Ghadiri M R 1998 J. Am. Chem. Soc. 12012108

[23] Lin V S Y, Motesharei K, Dancil K P S, Sailor M J and Reza Ghadiri M 1997 Science 278840

[24] Khaldun Salman A, Hassan Z and Khalid Omar 2012 Int. J. Electrochem. Sci. 7376

[25] Aspnes D E, Theeten J B and Hottier F 1979 Phys. Rev. B 20 3292

[26] Pickering C, Beale M I J, Robbins D J, Pearson P J and Greef R F 1985 Thin Solid Films 125157

[27] Pickering C, Beale M I J, Robbins D J, Pearson P J and Greef R F 1984 J. Phys. C 176535

[28] Tsu R, Shen H and Dutta M 1992 Appl. Phys. Lett. 60112

[29] Bisi O, Ossicini S and Pavesi L 2000 Surf. Sci. Rep. 381

[30] Chen C H and Chen Y F 1999 Solid State Commun. 111 681

[31] Qin G G and Jia Y Q 1993 Solid State Commun. 86559

[32] Prabakaran R, Kesavamoorthy R and Singh Alok 2005 Bull. Mater. Sci. 28219

[33] Xu H J and Li X J 2008 Opt. Express 162933 